\title{
ASYMPTOTIC STABILITY IN THE DISTRIBUTION OF NONLINEAR STOCHASTIC SYSTEMS WITH SEMI-MARKOVIAN SWITCHING
}

\author{
ZHENTING HOU', HAILING DONG ${ }^{\curvearrowleft}$ and PENG SHI ${ }^{2}$
}

(Received March 17, 2007; revised August 6, 2007)

\begin{abstract}
In this paper, finite phase semi-Markov processes are introduced. By introducing variables and a simple transformation, every finite phase semi-Markov process can be transformed to a finite Markov chain which is called its associated Markov chain. A consequence of this is that every phase semi-Markovian switching system may be equivalently expressed as its associated Markovian switching system. Existing results for Markovian switching systems may then be applied to analyze phase semi-Markovian switching systems. In the following, we obtain asymptotic stability for the distribution of nonlinear stochastic systems with semi-Markovian switching. The results can also be extended to general semi-Markovian switching systems. Finally, an example is given to illustrate the feasibility and effectiveness of the theoretical results obtained.
\end{abstract}

2000 Mathematics subject classification: primary 34A34; secondary $60 \mathrm{~K} 15$.

Keywords and phrases: phase distribution, semi-Markovian switching, associated Markov chain, asymptotic stability in distribution.

\section{Introduction}

In engineering applications, some frequently occurring dynamical systems which can be described by different forms depending on the value of an associated Markov chain process are termed Markovian switching systems. Research into this class of systems and their applications spans several decades. For some representative work on this general topic, we refer the reader to $[1-5,7-10,13-15,17]$ and the references therein. Some recent results can be found in $[16,18,19]$. Because the sojourn time at any state of a Markov chain is exponential, Markovian switchings have limited applications, and

\footnotetext{
'School of Mathematics, Central South University, Changsha, 410075 Hunan, China; email: hailing_fly@mail.csu.edu.cn.

${ }^{2}$ Faculty of Advanced Technology, University of Glamorgan, Pontypridd CF37 1DL, UK

(C) Australian Mathematical Society 2007, Serial-fee code 1446-1811/07
} 
the results obtained for these systems are conservative in some sense. In this paper, finite phase semi-Markov processes are introduced which require that the sojourn time at a state follows the phase distribution. As is well known, phase distributions are particularly dense, so research into phase semi-Markovian switching systems is significant. At first, we attempted to extend the results of Markovian switching systems to semi-Markovian switching systems. However it proved to be extremely difficult to do this directly. We therefore turned our attention to phase distributions which can approach all general distributions and are closely related to exponential distributions. Thus, we concentrate on considering phase semi-Markovian switching systems.

In Section 2, we first introduce the phase distribution and define phase semi-Markov processes. Then by introducing variables and a simple transformation, every finite phase semi-Markov process can be transformed to a finite Markov chain which is called its associated Markov chain. Then every phase semi-Markovian switching system may be equivalently expressed as its associated Markovian switching system. Existing results for Markovian switching systems may then be applied to analyze phase semi-Markovian switching systems. Furthermore, the family of phase distributions is particularly dense, so the results can also be extended to general semi-Markovian switching systems.

In Section 3, we apply our results to nonlinear stochastic differential equations and obtain Theorem 3.1. Then by Theorem 3.1 and the results in [18], we can obtain two criteria for asymptotic stability in the distribution of nonlinear stochastic systems with semi-Markovian switching. In fact, the results in [18] are for Markovian switching which required that transition times be distributed according to an exponential distribution. Our paper considers phase semi-Markovian switching. Furthermore, every general distribution can be approximated by a phase distribution. So our results can also be extended to general semi-Markovian switching systems. In fact, the ideas of this paper aren't limited to [18]. Almost all the nice results obtained so far on Markovian switching systems, for example, those of Ji and Chizeck [7], Costa et al. [1] and so on, are all valid for semi-Markovian switching systems. In Section 4, an example is given to illustrate the feasibility and effectiveness of the theoretic results obtained.

\section{Phase semi-Markov processes and Markovization}

Consider a continuous time Markov chain $a(t)$ on the state space $\{1,2, \cdots, m+1\}$, where the states $1,2, \cdots, m$ are transient and the state $m+1$ is absorbing. The infinitesimal generator is $Q=\left(\begin{array}{cc}T & T^{0} \\ 0 & 0\end{array}\right)$, where the $m \times m$ matrix $T=\left(T_{i j}\right)$ satisfies $T_{i i}<0, T_{i j} \geq 0, i \neq j, T^{0}=\left(T_{1}^{0}, T_{2}^{0}, \cdots, T_{m}^{0}\right)^{T}$ is a non-negative column vector, and $T e+T^{0}=0$. The initial distribution vector is $\left(\mathbf{a}, a_{m+1}\right)$, where $\mathbf{a}=\left(a_{1}, a_{2}, \cdots, a_{m}\right)$, $\mathbf{a} e+a_{m+1}=1$, and $e$ denotes an $m$-dimension column vector having $1 \mathrm{~s}$ as its compo- 
nents.

PROPOSITION 2.1 ([11]). The distribution of the time at which $a(t)$ is absorbed in $m+1$ is

$$
F(t)=1-\mathbf{a} \exp (T t) e, \quad t \geq 0
$$

DEFINITION 2.2 ([11]). The state that a $(t)$ reaches at time $t$ is called the phase of the distribution $F(\cdot)$ at time $t$.

DEFINITION 2.3 ([11]). The distribution $F(\cdot)$ defined in $(2.1)$ on $[0,+\infty)$ is called a phase $(P H)$ distribution and $(\mathbf{a}, T)$ is called its representation of order $m$.

There are many PH distributions, for example, a negative exponential distribution is a continuous $\mathrm{PH}$ distribution, and a $k$ th-order Erlang distribution $E$ is also a continuous $k$ PH distribution.

Furthermore, the $\mathrm{PH}$ distribution is very important because it has the following property.

PROPOSITION 2.4 ([11]). The family of $P H$ distribution is dense in all the families of distributions on $[0,+\infty)$.

In other words, for every probability distribution on $[0,+\infty)$, we may choose a PH distribution to approach the original distribution to any accuracy.

REMARK 1. A PH distribution is the distribution of an absorbing time in a finitestate Markov process. In other words, a phase random variable can be expressed as the time until absorption of a finite-state Markov chain with a single absorbing state. In 1954 Jensen [6] first introduced this distribution in an economics model, but he did not offer a useful method to deal with it. The key to making a PH distribution into a powerful tool is the matrix-analysis method developed by Neuts [12] in 1975. Since 1960s the PH distribution has been an important tool in stochastic models in queueing theory, storage theory, reliability theory, and so forth, and it has replaced the special status of the negative exponential distribution. But a $\mathrm{PH}$ distribution is still relatively unknown in many areas of science and engineering. Very recently, it has been shown that by virtue of the phase-type semi-Markov processes defined below, the PH distribution has important applications in control theory, Markov decision theory, Markov games and stochastic differential equations. We believe that there will be more and more applications of the PH distribution in the near future. In the present paper we will further show the application of the PH distribution in control theory. 
DEFINITION 2.5. Let $E$ be a finite set. A stochastic process $\hat{r}(t)$ on the state space $E$ is called a finite phase semi-Markov process, if it satisfies the following conditions:

(1) The sample paths of $(\hat{r}(t), t<+\infty)$ are right-continuous step functions and have left-hand limits with probability one.

(2) Denote the nth jump point of the process $\hat{r}(t)$ by $\tau_{n}(n=0,1,2, \cdots)$, where $\tau_{0} \equiv 0<\tau_{1}<\tau_{2}<\cdots<\tau_{n}<\cdots, \tau_{n} \uparrow+\infty$, and let the process $\hat{r}(t)$ have the Markov property at $\tau_{n}(n=0,1,2, \cdots)$. Then $\tau_{n}(n=0,1,2, \cdots)$ are Markov times of the process $\hat{r}(t)$.

(3) $F_{i j}(t)=P\left(\tau_{n+1}-\tau_{n} \leq t \mid r\left(\tau_{n}\right)=i, r\left(\tau_{n+1}\right)=j\right)=F_{i}(t)(i, j \in E, t \geq 0)$ is independent of $j$ and $n$.

(4) $F_{i}(t)(i \in E)$ is a phase distribution.

REMARK 2. When $F_{i}(t)(i \in E)$ is a negative exponential distribution, the finite phase semi-Markov process is a finite state Markov chain. A finite phase semiMarkov process enables us to overcome the restriction on the sojourn time at a state. Furthermore, by Proposition 2.4, for every probability distribution on $[0,+\infty)$, we may choose a PH distribution to approach the original distribution to any accuracy. However, key issues are how to deal with a finite phase semi-Markovian system and whether a finite phase semi-Markov process can be transformed to a finite Markov chain. We will show in the following that this transformation is possible by introducing variables and a simple transformation which is called Markovization.

Let $E$ be a finite nonempty set and let $\hat{r}(t)$ be a finite phase semi-Markov process on the state space $E$. Denote the $n$th jump point of the process $\hat{r}(t)$ by $\tau_{n}(n=0,1,2, \cdots)$, where $\tau_{0} \equiv 0<\tau_{1}<\tau_{2}<\cdots<\tau_{n}<\cdots$. Let $\left(\mathbf{a}^{(i)}, T^{(i)}\right)(i \in E)$ denote the $m^{(i)}$ order representation of $F_{i}(t)$, where $m^{(i)}$ is the number of the phase of $F_{i}(t)$. Let

$$
\begin{aligned}
F_{i}(t) & =P\left(\tau_{n+1}-\tau_{n} \leq t \mid \hat{r}\left(\tau_{n}\right)=i\right) \quad(i \in E), \\
\mathbf{a}^{(i)} & =\left(a_{1}^{(i)}, a_{2}^{(i)}, \cdots, a_{m^{(i)}}^{(i)}\right) \quad \text { and } \\
T^{(i)} & =\left(T_{j k}^{(i)}, j, k \in E\right) .
\end{aligned}
$$

Also let

$$
\begin{aligned}
p_{i j} & =P\left(\hat{r}\left(\tau_{n+1}\right)=j \mid \hat{r}\left(\tau_{n}\right)=i\right) \quad(i, j \in E), \\
P & =\left(p_{i j}, i, j \in E\right) \quad \text { and } \\
(\mathbf{a}, T) & =\left\{\left(\mathbf{a}^{(i)}, T^{(i)}\right), \quad i \in E\right\}
\end{aligned}
$$

Obviously, the probability distribution of $\hat{r}(t)$ can be determined only by $\{P,(\mathbf{a}, T)\}$.

DEFINITION 2.6. The term $\{P,(\mathbf{a}, T)\}$ is called the pair of a finite phase semiMarkov process $\hat{r}(t)$. 
For every $n(n=0,1, \cdots), \tau_{n} \leq t<\tau_{n+1}$, define

$$
J(t)=\text { the phase of } F_{\hat{r}(t)}(\cdot) \text { at time } t-\tau_{n} .
$$

DEFINITION 2.7. The parameter $J(t)$ defined in (2.5) is called the phase of $\hat{r}(t)$ at time $t$.

For any $i \in E$, we define

$$
\begin{aligned}
T_{j}^{(i, 0)} & =1-\sum_{k=1}^{m(i)} T_{j k}^{(i)} \quad\left(j=1,2, \cdots, m^{(i)}\right), \\
G & =\left\{\left(i, k^{(i)}\right) \mid i \in E, k^{(i)}=1,2, \cdots, m^{(i)}\right\} .
\end{aligned}
$$

Our first main result in this paper is as follows.

THEOREM 2.8. The variable $Z(t)=(\hat{r}(t), J(t))$ is a Markov chain with state space $G$, where $G$ is finite if and only if $E$ is finite. The infinitesimal generator of $Z(t)$ given by $Q=\left(q_{\mu \nu}, \mu, \nu \in G\right)$ is determined only by the pair of $\hat{r}(t)$ given by $\{P,(a, T)\}$ as follows:

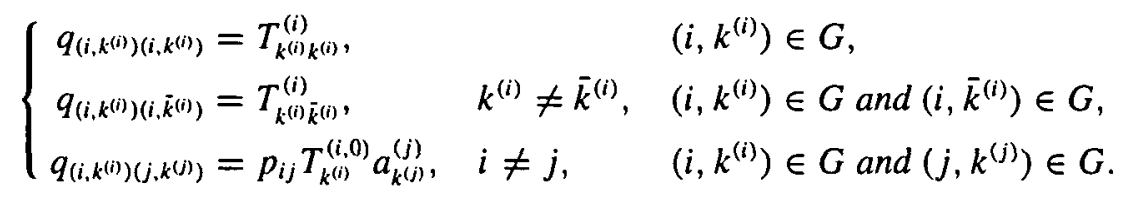

PROOF. 1) For every $n(n=0,1, \cdots), \tau_{n} \leq t<\tau_{n+1}$, define $J(t)$ to be the phase of $F_{\hat{r}(t)}(\cdot)$ at time $t-\tau_{n}$, which gives the information of $\hat{r}(t)$ on $\left(\tau_{n}, t\right]$, so by supplying this variable, $Z(t)=(\hat{r}(t), J(t))$ is a Markov chain.

2) Assume that $E$ has finite elements, then $G$ has $s=\sum_{i \in E} m^{(i)}$ elements, where $\hat{r}(t)$ is as in Definition 2.5. Hence the state space of $Z(t)$ has finite elements.

3) As for (2.7), when $\left(i, k^{(i)}\right) \in G$, there is no change of state and phase, so $q_{\left(i, k^{(i)}\right)\left(i, k^{(i)}\right)}=$ $T_{k^{(i)} k^{(i)}}^{(i)}$;

when $\left.k^{(i)} \neq \bar{k}^{(i)}, i, k^{(i)}\right) \in G$ and $\left(i, \bar{k}^{(i)}\right) \in G$, there is no change of state while there is a change of phase, so $q_{\left(i, k^{(i)}\right)\left(i, \bar{k}^{(i)}\right)}=T_{k^{(i)} \bar{k}^{(i)}}^{(i)}$;

when $i \neq j,\left(i, k^{(i)}\right) \in G$ and $\left(j, k^{(j)}\right) \in G$, there are changes of state and phase. According to (2.2) and (2.6), we will have $q_{\left(i, k^{(i)}\right)\left(j, k^{(j)}\right)}=p_{i j} T_{k^{(i)}}^{(i .0)} a_{k^{(j)}}^{(j)}$.

Then the theorem is proved.

By Theorem 2.8, we will now construct the associated Markov chain of $\hat{r}(t)$.

Assume that $G$ has $s=\sum_{i \in E} m^{(i)}$ elements, so that the state space of $Z(t)$ has $s$ elements. We number the $s$ elements according to the following method. Denote the number of $(i, k)$ by $\sum_{r=1}^{i-1} m^{(r)}+k\left(1 \leq k \leq m^{(i)}\right)$. Denote this transformation by $\varphi$, 
hence we get

$$
\varphi((i, k))=\sum_{r=1}^{i-1} m^{(r)}+k \quad\left(i \in E, 1 \leq k \leq m^{(i)}\right) .
$$

We define

$$
\alpha_{\varphi((i, k)) \varphi\left(\left(^{\prime}, k^{\prime}\right)\right)}=q_{(i, k)\left(i^{\prime}, k^{\prime}\right)} \quad \text { and } \quad r(t)=\varphi(Z(t))
$$

where $r(t)$ is called the associated Markov chain of $\hat{r}(t)$.

According to Theorem 2.8, $Z(t)$ is a Markov chain, so $r(t)$ is a Markov chain with state space $S=\{1,2, \cdots, s\}$ and infinitesimal generator $Q=\left(\alpha_{i m}, 1 \leq i, m \leq s\right)$.

By now, we know that every finite phase semi-Markov process can be transformed to a finite Markov chain which is called its associated Markov chain. Then every phase semi-Markovian switching system may be equivalently expressed as its associated Markovian switching system. In the following, we apply the results to a class of nonlinear stochastic differential equations.

\section{Applying the results to nonlinear stochastic differential equations}

Let $\{\hat{r}(t), t \geq 0\}$ be a finite phase semi-Markov process with a state space $E$. Then the probability distribution of $\hat{r}(t)$ can be determined by $\{P,(\mathbf{a}, T)\}$ as defined in (2.3) and (2.4). Consider a class of nonlinear stochastic differential equations with semi-Markovian switching in a fixed probability space $(\Omega, \mathscr{F}, P)$ :

$$
d \mathbf{X}(t)=\hat{f}(\mathbf{X}(t), \hat{r}(t)) d t+\hat{g}(\mathbf{X}(t), \hat{r}(t)) d B(t), \quad t \geq 0,
$$

where $\hat{r}(0)=r_{0}$, on $t=0$, with initial state $\mathbf{X}(0)=x_{0}$, where

$$
\hat{f}: R^{n} \times S \rightarrow R^{n}, \quad \hat{g}: R^{n} \times S \rightarrow R^{n \times m} \text {. }
$$

Then we have our second result.

THEOREM 3.1. As for system (3.1), we can construct another system as follows such that it is equivalent to (3.1):

$$
d X(t)=f(X(t), r(t)) d t+g(X(t), r(t)) d \boldsymbol{B}(t), \quad t \geq 0
$$

where $r(t)$ is the associated Markov chain of $\hat{r}(t)$. Then system (3.2) is called the associated Markovian switching system of (3.1).

PROOF. First we try to find the associated Markov chain $r(t)$. According to Theorem 2.8, $\mathcal{Z}(t)=(\hat{r}(t), J(t))$ is a Markov chain with state space $G$. The generator of $Z(t)$ is given by $Q=\left(q_{\mu \nu}, \mu, \nu \in G\right)$ which is determined only by the pair 
of $\hat{r}(t)$ given by $\{P,(\mathbf{a}, T)\}$. Then by constructing the associated Markov chain of $\hat{r}(t)$, we know that $r(t)=\varphi(Z(t))$ is the associated Markov chain with state space $S=\{1,2, \cdots, s\}$ and infinitesimal generator $Q=\left(\alpha_{i m}, 1 \leq i, m \leq s\right)$. For any $\alpha \in R^{n}, i \in E, k=1,2, \cdots, m^{(i)}$, we construct functions $f, g$ as follows:

$$
\begin{aligned}
& f(\alpha, \varphi(i, k))=f\left(\alpha, \sum_{r=1}^{i-1} m^{(r)}+k\right) \triangleq \hat{f}(\alpha, i), \quad k=1,2, \cdots, m^{i}, \quad \text { and } \\
& g(\alpha, \varphi(i, k))=g\left(\alpha, \sum_{r=1}^{i-1} m^{(r)}+k\right) \triangleq \hat{g}(\alpha, i), \quad k=1,2, \cdots, m^{i} .
\end{aligned}
$$

Then system (3.2) is constructed. By the construction, we know it is equivalent to (3.1). The proof is completed.

REMARK 3. According to Theorem 3.1, if we want to investigate asymptotic stability in the distribution of the phase semi-Markovian switching system (3.1), we investigate its associated Markovian switching system (3.2).

Note that system (3.2) has been studied in [18]. By use of Theorem 3.1 and the results in [18], we can obtain the following results.

THEOREM 3.2. If $f$ and $g$ in (3.2) satisfy a local Lipschitz condition and a linear growth condition, furthermore Equation (3.2) has the following properties:

(P1) For any $(x, i) \in R^{n} \times S$ and any $\varepsilon>0$, there exists a constant $R>0$ such that

$$
P\left\{\left|X^{x, i}(t) \geq R\right|\right\}<\varepsilon \quad \forall t \geq 0 .
$$

(P2) For any $\varepsilon>0$, and any compact subset $K$ of $R^{n}$, there exists a $T=T(\varepsilon, K)>0$ such that

$$
P\left\{\left|X^{x, i}(t)-X^{y, i}(t)<\varepsilon\right|\right\} \geq 1-\varepsilon, \quad \forall t \geq T,
$$

where, by $X^{x, i}(t)$ we denote the solution of Equation (3.2) with initial conditions $X(0)=x \in R^{n}$ and $r(0)=i$. Here $S$ is the state space of the associated Markov chain $r(t)$. Then Equation (3.1) is asymptotically stable in distribution.

PROOF. First from [18], under a local Lipschitz condition and a linear growth condition, if system (3.2) has the properties (P1) and (P2), system (3.2) is asymptotically stable in distribution. According to Theorem 3.1, system (3.1) is equivalent to the associated Markovian switching system (3.2), so system (3.1) is asymptotically stable in distribution. The proof ends. 
To make our theory more applicable, let us now use the results obtained previously to develop a new criterion in terms of M-matrices, which can be verified easily in practice.

THEOREM 3.3. If $f$ and $g$ in (3.2) satisfy a local Lipschitz condition and a linear growth condition, further assume that for all $x, y \in R^{n}$ and $i \in S$,

$$
\left\{\begin{array}{l}
x^{T} f(x, i) \leq \beta_{i}|x|^{2}+\gamma \\
(x-y)^{T}(f(x, i)-f(y, i)) \leq \beta_{i}|x-y|^{2}, \\
|g(x, i)|^{2} \leq \delta_{i}|x|^{2}+\gamma \\
|g(x, i)-g(y, i)|^{2} \leq \delta_{i}|x-y|^{2}
\end{array}\right.
$$

where $\gamma, \beta_{i}$ and $\delta_{i}$ are constants. If

$$
\Lambda:=-\operatorname{diag}\left(2 \beta_{1}+\delta_{1}, 2 \beta_{2}+\delta_{2}, \ldots 2 \beta_{N}+\delta_{N}\right)-Q
$$

is an M-matrix, where $S$ and $Q$ are the state space and infinitesimal generator of the associated Markov chain $r(t)$ separately, then system (3.1) is asymptotically stable in distribution.

PROOF. First from [18], if $f$ and $g$ in (3.2) satisfy a local Lipschitz condition, a linear growth condition and (3.3), and furthermore, if (3.4) is an M-matrix, then system (3.2) is asymptotically stable in distribution. According to Theorem 3.1, system (3.1) is equivalent to its associated Markovian switching system (3.2), so system (3.1) is asymptotically stable in distribution, which completes the proof.

REMARK 4. Almost all the nice results obtained so far on Markovian switching systems, for example, those of $\mathrm{Ji}$ et al. ([7-9]), Shi et al. ([13-16]) and Costa et al. ([1]), and the references therein, are all valid on phase semi-Markovian switching systems.

\section{Example}

Let $\hat{r}(t)$ be a phase semi-Markov process taking values in $S=\{1,2\}$. The sojourn time in the first state is a random variable distributed according to a negative exponential distribution with parameter $\lambda_{1}$. The sojourn time in the second state is a a random variable distributed according to a 2-order Erlang distribution.

Let $B(t)$ be a scalar Brownian motion and let $\hat{r}(t)$ be defined as above. Assume that $B(t)$ and $\hat{r}(t)$ are independent. Consider a one-dimensional stochastic differential equation with phase type semi-Markovian switching

$$
d X(t)=\hat{\alpha}(\hat{r}(t)) X(t) d t+\sigma d B(t), \quad t \geq 0
$$


where $\sigma$ is a constant, $\hat{\alpha}(1)=1$ and $\hat{\alpha}(2)=-1 / 2$.

This system is not a Markovian switching system but a phase semi-Markovian switching system. In the past, we have had no method to deal with phase semiMarkovian switching. But by Theorem 3.1 in this paper, we know if we want to investigate a phase semi-Markovian switching system, we can investigate its associated Markovian switching system. The key is looking for the associated Markov chain and its infinitesimal generator, and defining the proper function.

In fact, the sojourn time in the second part can be divided into two parts. The sojourn time in the first (respectively second) subdivision is a random variable that is negative exponentially distributed with parameter $\lambda_{2}$, (respectively $\lambda_{3}$ ). More particularly, if the process $\hat{r}(t)$ enters State 2, it must first stay at the first subdivision for some time, then enter the second subdivision, then return to State 1 again.

We know that $p_{12}=p_{21}=1$. Obviously,

$$
\begin{gathered}
\left(\begin{array}{ll}
p_{11} & p_{12} \\
p_{21} & p_{22}
\end{array}\right)=\left(\begin{array}{ll}
0 & 1 \\
1 & 0
\end{array}\right), \quad \mathbf{a}^{(1)}=\left(a_{1}^{(1)}\right)=(1), \quad \mathbf{a}^{(2)}=\left(a_{1}^{(2)}, a_{2}^{(2)}\right)=(1,0), \\
T^{(1)}=\left(T_{11}^{(1)}\right)=\left(-\lambda_{1}\right) \quad \text { and } \quad T^{(2)}=\left(\begin{array}{ll}
T_{11}^{(2)} & T_{12}^{(2)} \\
T_{21}^{(2)} & T_{22}^{(2)}
\end{array}\right)=\left(\begin{array}{cc}
-\lambda_{2} & \lambda_{2} \\
0 & -\lambda_{3}
\end{array}\right) .
\end{gathered}
$$

It is easy to see the state space of $Z(t)=(\hat{r}(t), J(t))$ is $G=((1,1),(2,1),(2,2))$. We number the elements of $G$ as $\varphi((1,1))=1, \varphi((2,1))=2$ and $\varphi((2,2))=3$. Hence, the infinitesimal generator of $\varphi(Z(t))$ is

$$
Q=\left(\alpha_{i j}\right)=\left(\begin{array}{ccc}
-\lambda_{1} & \lambda_{1} & 0 \\
0 & -\lambda_{2} & \lambda_{2} \\
\lambda_{3} & 0 & -\lambda_{3}
\end{array}\right)
$$

Now, let $r(t)=\varphi(Z(t))$. It is obvious that $r(t)$ is the associated Markov chain of $\hat{r}(t)$ with state space $\{1,2,3\}$. The infinitesimal generator of $r(t)$ is given by (4.2).

For to the construction of $f$, we have that

$$
\alpha(1)=\hat{\alpha}(1) \quad \text { and } \quad \alpha(1+k)=\hat{\alpha}(k), \quad k=1,2 .
$$

Then, by Theorem 3.1, system (4.1) is equivalent to the following system:

$$
\left\{\begin{array}{l}
d X(t)=X(t) d t+\sigma d B(t) \\
d X(t)=-\frac{1}{2} X(t) d t+\sigma d B(t) \\
d X(t)=-\frac{1}{2} X(t) d t+\sigma d B(t)
\end{array}\right.
$$

for state 1 and phases 1 and 2 of state 2 respectively. 
From a property of the Ornstein-Uhlenbeck process, we observe that the first equation in system (4.1) is not asymptotically stable in distribution. However, in the following, it can be seen that if the parameters $\left(\lambda_{1}, \lambda_{2}, \lambda_{3}\right)$ of phase semi-Markovian switching satisfy some condition, the overall system (4.1) will be asymptotically stable in distribution which precisely reflects the importance of phase semi-Markovian switching systems.

In fact, by the definition of $\alpha$, it can be easily verified that a local Lipschitz condition and a linear growth condition hold, and condition (3.3) applies with

$$
\beta_{1}=1, \quad \beta_{2}=-\frac{1}{2}, \quad \beta_{3}=-\frac{1}{2}, \quad \delta_{1}=\delta_{2}=\delta_{3}=0 \quad \text { and } \quad \gamma=\sigma^{2} .
$$

So the matrix defined by (3.4) becomes

$$
\Lambda:=-\operatorname{diag}(2,-1,-1)-Q=\left(\begin{array}{ccc}
-2+\lambda_{1} & -\lambda_{1} & 0 \\
0 & 1+\lambda_{2} & -\lambda_{2} \\
-\lambda_{3} & 0 & 1+\lambda_{3}
\end{array}\right) .
$$

This is an M-matrix if and only if

$$
\left\{\begin{array}{l}
-2+\lambda_{1}>0 \\
\left(-2+\lambda_{1}\right)\left(1+\lambda_{2}\right)>0 \\
\left(-2+\lambda_{1}\right)\left(1+\lambda_{2}\right)\left(1+\lambda_{3}\right)+\left(-\lambda_{1}\right)\left(-\lambda_{2}\right)\left(-\lambda_{3}\right)>0 .
\end{array}\right.
$$

That is,

$$
\lambda_{1}>2 \text { and }\left(\lambda_{1}-2\right)\left(1+\lambda_{2}+\lambda_{3}\right)-2 \lambda_{2} \lambda_{3}>0 .
$$

By Theorem 3.3, we can therefore conclude that system (4.1) is asymptotically stable in distribution if

$$
\lambda_{1}>2 \text { and }\left(\lambda_{1}-2\right)\left(1+\lambda_{2}+\lambda_{3}\right)-2 \lambda_{2} \lambda_{3}>0 .
$$

\section{Conclusions}

The new and original ideas of this paper are Markovization by supplying variables and considering a phase distribution. By the methods of supplying variables and making a simple transformation, we proved that every finite phase semi-Markov process can be transformed to a finite Markov chain which is called its associated Markov chain. Then we can obtain that every phase semi-Markov switching system may be equivalently expressed as its associated Markov switching system. Existing results for Markovian switching systems may then be applied to analyze phase semiMarkovian switching systems. The key is looking for the associated Markov chain and its infinitesimal generator. We can then use the results of Markov switching to obtain the desired results. A example is given to illustrate the feasibility and effectiveness of the theoretic results obtained. 


\section{Acknowledgements}

This work was supported by the National Natural Science Foundation of China (Grant No.10671212), the Hi-Tech Research and Development Program of China (Grant No.20050533036) and the PhD paper innovation fund (Grant No.3340-76206). The authors would like to thank the editor and the anonymous reviewers for their constructive comments and suggestions for improving the quality of the paper.

\section{References}

[1] O. L. V. Costa and M. D. Fragoso, "Stability results for discrete-time linear systems with Markovian jumping parameters", J. Math. Anal. Appl. 179 (1993) 154-178.

[2] M. Davis, Markov models and optimization (Chapman and Hall, London, 1992).

[3] R. J. Elliott and D. D. Sworder, "Control of a hybrid conditionally linear Gaussian processes", $J$. Optim. Theory Appl. 74 (1992) 75-85.

[4] X. Feng, K. A. Loparo, Y. Ji and H. J. Chizeck, "Stochastic stability properties of jump linear systems", IEEE Trans. Automat. Control 37 (1992) 38-53.

[5] W. Fleming, S. Sethi and M. Soner, "An optimal stochastic production planning problem with randomly fluctuating demand", SIAM J. Control Optim. 25 (1987) 1494-1502.

[6] A. Jensen, A distribution model applicable to economics (Munksgaard, Copenhagen, 1954).

[7] Y. Ji and H. J. Chizeck, "Controllability, stabilizability and continuous-time Markovian jump linear-quadratic control", IEEE Trans. Automat. Control 35 (1990) 777-788.

[8] Y. Ji and H. J. Chizeck, "Jump linear quadratic Gaussian control: steady-state solution and testable conditions", Control Theory Adv. Tech. 6 (1990) 289-319.

[9] Y. Ji, H. J. Chizeck, X. Feng and K. A. Loparo, "Stability and control of discrete time jump linear systems", Control Theory and Advanced Technology 7 (1991) 247-270.

[10] H. Kushner, Stochastic stability and control (Academic Press, New York-London, 1967).

[11] M. F. Neuts, "Probability distributions of phase type", in Liber Amicorum Prof. Belgium Univ. of Louvain, (1975) 173-206.

[12] M. F. Neuts, Matrix-geometric solutions in stochastic models (John Hopkins Univ. Press, Baltimore, Md., 1981).

[13] P. Shi and E. K. Boukas, " $H_{\infty}$ control for Markovian jumping linear systems with parametric uncertainty", J. Optim. Theory Appl. 95 (1997) 75-99.

[14] P. Shi, E. K. Boukas and R. K. Agarwal, "Control of Markovian jump discrete-time systems with norm bounded uncertainty and unknown delays", IEEE Trans. Automat. Control 44 (1999) 2139-2144.

[15] P. Shi, E. K. Boukas and R. K. Agarwal, "Kalman filtering for continuous-time uncertain systems with Markovian jumping parameters", IEEE Trans. Automat. Control 44 (1999) 1592-1597.

[16] P. Shi, E. K. Boukas, S. K. Nguang and X. Guo, "Robust disturbance attenuation for discrete-time active fault tolerant control systems with uncertainties", Optimal Control Appl. Methods 24 (2003) 85-101.

[17] A. V. Skorohod, Asymptotic methods in the theory of stochastic differential equations (American Mathematical Society, Providence, RI, 1989).

[18] C. Yuan and X. Mao, "Asymptotic stability in distribution of stochastic differential equations with Markovian switching", Stochastic Process. Appl. 103 (2003) 277-291.

[19] C. Yuan, J. Zhou and X. Mao, "Stability in distribution of stochastic differential delay equations with Markovian switching", System Control Lett. 50 (2003) 277-291. 